KODDAI MATH. SEM. RFP.

19 (1967), 61-74

\title{
ASYMPTOTICALLY MOST INFORMATIVE PROCEDURE IN THE CASE OF EXPONENTIAL FAMILIES
}

\author{
By Kazutomo Kawamura
}

\section{§ 1. Introduction.}

Recently we showed the following fact in our paper [2]. We considered in [2] two binomial trials $E_{1}, E_{2}$ having unknown means $p_{1}, p_{2}$ respectively. And we have introduced the notion of costs such that we must pay costs $c_{1}, c_{2}$ to the observation of a result given by the trials $E_{1}, E_{2}$ respectively. In each step we are admitted to select one of the two trials $E_{1}, E_{2}$. Be continued the selections by some way we denoted the sequence of trials till $n$-th step as $E^{(1)}, \cdots, E^{(n)}$ and the sequence of costs till $n$-th step as $C^{(1)}, \cdots, C^{(n)}$. Of course we may select at $i$-th step $E^{(i)}$ from the two trials $E_{1}, E_{2}$ depending previous $i-1$ data $X_{1}, \cdots, X_{\imath-1}$ given by $E^{(1)}, \cdots, E^{(i-1)}$. A procedure $\mathscr{L}$ was given in [2] such that the sum of information given by two dimensional likelihood ratio relative to the sum of costs till $n$-th step to discriminate $p_{1}>p_{2}$ or $p_{1}<p_{2}$ is asymptotically maximized. In [2] we assumed the unknown true two dimensional parameter $\left(p_{1}, p_{2}\right)$ did not exist on the boundary $p_{1}=p_{2}$. In our another paper [3] we considered analogous model having two kinds of trials $E_{1}, E_{2}$ which are obeyed normal distributions with unknown means $m_{1}, m_{2}$ and known same variance $\sigma^{2}$ and costs $c_{1}, c_{2}$ respectively. Then analogous procedure $\mathscr{L}$ is asymptotically optimal in the same sense described above. In [3] we noted that our procedure $\mathscr{Q}$ reduced to a policy which does not depending on previous $n$ data $X_{1}, \cdots, X_{n}$ but only on sample sizes $n_{1}$ of $E_{1}, n_{2}$ of $E_{2}$ till $n$-th step. We have omitted the proof of the problem in [3] because we can easily get analogous proof.

In this paper we generalize these problems to $k$ trials $E_{1}, \cdots, E_{k}$ having exponential distributions with one dimensional unknown parameter $\theta_{1}, \cdots, \theta_{k}$ respectively. That is, an observation $X$ of $E_{\jmath}$ has a probability density function of exponential type in Kullback's sense [4] with one dimensional unknown parameter $\theta_{j}(j=1, \cdots, k)$ respectively. And we introduced the boundary $\pi: \mu \cdot \theta=p\left(\theta=\left(\theta_{1}, \cdots, \theta_{k}\right)\right)$ as a hyperplane in $k$ dimensional euclidean space where $\mu=\left(\mu_{1}, \cdots, \mu_{k}\right)$ is any fixed $k$ dimensional unit vector having all non-zero components and $p$ is any fixed nonnegative number and $\mu \cdot \theta$ is the inner product of two vectors $\mu$ and $\theta$. Moreover we use the notion of costs introduced by Kunisawa [6], as we used the notion in [2], [3], then we can get some information of $\theta_{j}$ by paying of cost $c_{j}(j=1, \cdots, k)$ respectively. Then we shall show analogously that under the generalized procedure $\mathscr{L}^{*}$ given in the following Section 3 the sum of information relative to the sum of costs payed till $n$-th step to discriminate $\mu \cdot \theta$ larger than $p$ or not is asymptotically

Received June 30, 1966. 
maximized. Additionally we show in this paper that under the original procedure $\mathscr{L}$ given in [2], [3] the ratio is also asymptotically maximized in the sense of the generalized procedure $\mathscr{L}^{*}$. Moreover the problem given in [3] will be shown in special example of case $k=2$ in Section 5. Finally note that in this paper we need not to assume that our unknown true parameter $\theta$ is not an element of our hyperplane $\pi$.

\section{$\S 2$. Notations, definitions and some lemmas.}

Definition of the exponential family introduced by S. Kullback. Suppose that $f\left(x, \theta_{0}\right)$ and $f\left(x, \theta_{1}\right)$ are generalized densities of a dominated set of probability measures on the measurable space $(\mathfrak{X}, \Phi)$ so that

$$
\nu_{i}(E)=\int_{E} f\left(x, \theta_{i}\right) d x \quad E \in \Phi,(i=0,1) .
$$

For a given $f\left(x, \theta_{0}\right)$ we seek the member of the dominated set of probability measures that is "nearest" to or most closely resembles the probability measure $\nu_{0}$ in the sense of smallest directed divergence

$$
I\left(\theta_{1}, \theta_{0}\right)=\int_{R} f\left(x, \theta_{1}\right) \log \frac{f\left(x, \theta_{1}\right)}{f\left(x, \theta_{0}\right)} d x .
$$

as a restriction of $f\left(x, \theta_{1}\right)$ we shall require $f\left(x, \theta_{1}\right)$ minimizing $I\left(\theta_{1}, \theta_{0}\right)$ subject to

$$
\int_{R} T(x) f\left(x, \theta_{1}\right) d x=\theta_{1}
$$

where $\theta_{1}$ is any fixed constant and $Y=T(x)$ a measurable statistic. Then the minimum value of $I\left(\theta_{1}, \theta_{0}\right)$ is given if and only if

$$
f\left(x, \theta_{1}\right)=e^{\tau\left(\theta_{1}\right) T(x)} f\left(x, \theta_{0}\right) / M\left(\tau\left(\theta_{1}\right)\right)
$$

where

$$
M\left(\tau\left(\theta_{1}\right)\right)=\int_{R} f\left(x, \theta_{0}\right) e^{\tau\left(\theta_{1}\right) T(x)} d x
$$

We remark that

$$
f(x, \theta)=f\left(x, \theta_{0}\right) e^{\tau(\theta) T(x)} / M(\tau(\theta))
$$

is said to generate an exponential family of distributions, the family of exponential type determined by $f(x, \theta)$, as $\theta$ ranges over its values satisfying $M(\tau(\theta))<\infty$.

$$
\theta=\frac{d}{d \tau} \log M(\tau(\theta)) \quad \text { and } \quad \nu_{0}(x: T(x)=\theta) \neq 1,
$$

then $\tau(\theta)$ is a strictly increasing function of $\theta$. Using this fact if $f\left(x, \theta_{1}\right)$ and $f\left(x, \theta_{2}\right)$ are the members of common exponential family generated by $f\left(x, \theta_{0}\right)$ then for any fixed $\theta$ in the interval $\left[\theta_{1}, \theta_{2}\right]\left(\theta_{1}<\theta_{2}\right)$ 


$$
\begin{aligned}
M(\tau(\theta)) & =\int_{R} f\left(x, \theta_{0}\right) e^{\tau(\theta) T(x)} d x \\
& \leqq \int_{T(x) \geqq 0} f\left(x, \theta_{0}\right) e^{\tau\left(\theta_{2}\right) T(x)} d x \\
& +\int_{T(x)<0} f\left(x, \theta_{0}\right) e^{\tau\left(\theta_{1}\right) T(x)} d x<\infty
\end{aligned}
$$

so that the exponential family is defined on connected interval or full line $R=(-\infty, \infty)$. Next we consider $n$ independent observations $x_{1}, \cdots, x_{n}$ from true density function $f(x, \theta)$ in the exponential family generated by $f\left(x, \theta_{0}\right)$. Then logarithm of the likelihood function $\Pi_{\imath=1}^{n} f\left(x_{\imath}, \theta\right)$ is expressed as follows.

$$
\log \prod_{i=1}^{n} f\left(x_{i}, \theta\right)=\log \prod_{i=1}^{n} f\left(x_{i}, \theta_{0}\right)+\tau(\theta) \sum_{i=1}^{n} T\left(x_{i}\right)-n \log M(\tau(\theta))
$$

The maximum value of $\Pi_{i=1}^{n} f\left(x_{i}, \theta\right)$ is given if and only if $\theta=\hat{\theta}_{n}$ as follows

$$
\begin{gathered}
\frac{d}{d \theta} \log \prod_{i=1}^{n} f\left(x_{i}, \theta\right)=0, \\
\frac{d \tau}{d \theta} \cdot\left\{\sum_{i=1}^{n} T\left(x_{i}\right)-n \frac{d}{d \tau} \log M(\tau(\theta))\right\}=0
\end{gathered}
$$

where $d \tau / d \theta>0$ is satisfied.

$$
\hat{\theta}_{n}=\frac{d}{d \tau} \log M(\tau(\theta))=\frac{\sum_{\imath=1}^{n} T\left(x_{\imath}\right)}{n} .
$$

In the following line we suppose that the true parameter $\theta$ is finite, then by the strong law of large numbers $\hat{\theta}_{n} \rightarrow \theta$ as $n \rightarrow \infty$ is satisfied with probability 1 .

Definition of parameter space $\Theta$. Our $k$ exponential families with one dimensional parameter $\theta_{j}(j=1, \cdots, k)$ are defined on one dimensional open intervals $\Theta_{\jmath}$ of $\theta$, respectively. Then our unknown parameter $\theta=\left(\theta_{1}, \cdots, \theta_{k}\right)$ is an element of $k$ dimensional parameter space

$$
\Theta=\Theta_{1} \otimes \cdots \otimes \Theta_{k}
$$

which is a product space of $k$ open intervals $\Theta_{1}, \cdots, \Theta_{k}$. Next we divide the space $\Theta$ by our hyperplane $\mu \cdot \theta=p$ as follows. Of course we assume the hyperplane acrosses our parameter space $\Theta$.

$$
\begin{gathered}
H_{1}=\{\theta: \mu \cdot \theta>p, \theta \in \Theta\}, \quad H_{2}=\{\theta: \mu \cdot \theta<p, \theta \in \Theta\}, \\
\pi=\{\theta: \mu \cdot \theta=p, \theta \in \Theta\} .
\end{gathered}
$$

Then $\Theta=H_{1}+H_{2}+\pi$ is satisfied. Next let $E^{(i)}$ be $i$-th trial which is one of $k$ elements $E_{1}, \cdots, E_{k}$ and define $X_{\imath}$ to be $i$-th random variable which is given by trial $E^{(i)}$ randomizedly.

Calculation of likelihood function of $\theta$. Given the first $n$ trials in some way, we define the number of $E_{\jmath}$ in $E^{(1)}, \cdots, E^{(n)}$ as $n_{\jmath}$. In the following line we 
suppose $n_{j} \geqq 1(j=1, \cdots, k)$ then all $n_{j}$ observations $X_{\imath}$ from the trial $E_{j}$ in the first $n$ trials $E^{(1)}, \cdots, E^{(n)}$ is considered as $n$, independent observations from the trial $E_{\jmath}$. Therefore the likelihood function of the parameter $\theta_{\jmath}$ is expressed by

$$
\prod_{\left\{i: E(i)=E_{j}\right\}} f\left(x_{i}, \theta, E_{j}\right)
$$

where $\left\{i: E^{(i)}=E_{\jmath}\right\}$ is a set of $n_{\jmath}$ elements of $i$ in $1, \cdots, n$ satisfying $E^{(i)}=E_{\jmath}$ for any fixed $j(j=1, \cdots, k)$. Then for given $E^{(1)}, \cdots, E^{(n)}$ the likelihood function of $\theta$ is expressed by the product as following

$$
\prod_{i=1}^{n} f\left(x_{i}, \theta, E^{(i)}\right)=\prod_{\left\{i: E(i)=E_{1}\right\}} f\left(x_{i}, \theta, E_{1}\right) \cdots \prod_{\left\{i: E(i)=E_{k}\right\}} f\left(x_{i}, \theta, E_{k}\right)
$$

Of course, for any fixed $E_{\jmath}$, the probability density function $f\left(x, \theta, E_{j}\right)$ of the trial $E_{\jmath}$ for every $\theta=\left(\theta_{1}, \cdots, \theta_{k}\right)$ in $\Theta$ is considered as a function of $\theta_{\jmath}$ only and independent of $\theta_{i}(i \neq j)$.

Definition of $\hat{\theta}_{n}$ and unique existence of the value. We denote as $\hat{\theta}_{n}$ the maximum likelihood estimate of $\theta$. The $j$-th component of $\hat{\theta}_{n}$ will maximize the $j$-th likelihood function

$$
\prod_{\left\{i: E(i)=E_{j}\right\}} f\left(x_{i}, \theta, E_{j}\right)
$$

with respect to $\theta_{\jmath}$. We denote the value of $\theta_{\jmath}$ which maximizes the $j$-th likelihood function as $\hat{\theta}_{n \jmath}$. Then $\hat{\theta}_{n \jmath}$ given by the $n_{\jmath}$ trials of $E_{\jmath}$ is uniquely expressed as followings from the discussion of exponential family

$$
\hat{\theta}_{n_{\jmath}}=\frac{\sum_{\left\{i: E(i)=E_{j}\right\}} T_{j}\left(x_{i}\right)}{n_{\jmath}}
$$

where $T_{j}(x)$ is a statistic of trial $E_{\jmath}$ satisfying

$$
\int_{R} T_{j}(x) f\left(x, \theta, E_{j}\right) d x=\theta_{\jmath} \quad(j=1, \cdots, k)
$$

as in (2.4). Hence our $\hat{\theta}_{n}$ is expressed as following, if $n_{j} \geqq 1 \quad(j=1, \cdots, k)$ is satisfied,

$$
\hat{\theta}_{n}=\left\{\hat{\theta}_{n 1}, \cdots, \hat{\theta}_{n k}\right\} .
$$

And the uniqueness of $\hat{\theta}_{n}$ is reduced to that of $\hat{\theta}_{n \jmath}$. So that we get the following lemma as to be proved.

LEMMA 1. Under any sequence $E^{(1)}, \cdots, E^{(n)}$ if $n_{j} \geqq 1(j=1, \cdots, k)$ is satisfied then there exists $\hat{\theta}_{n}$ uniquely on our parameter space $\Theta$.

Definition of $\tilde{\theta}_{n}$. Next we shall denote by $\tilde{\theta}_{n}$ the maximum likelihood estimate of $\theta$ on the subspace $a\left(\hat{\theta}_{n}\right)$ over the first $n$ trials where $a(\theta)$ is defined as follows:

$$
\begin{aligned}
& a(\theta)=\Theta-H_{i} \quad \text { if } \quad \theta \in H_{\imath} \quad(i=1,2), \\
& =\Theta \quad \text { if } \quad \theta \in \pi \text {. }
\end{aligned}
$$


Definition of sum of information. Now we define the sum of information to discriminate $\mu \cdot \theta>p$ or not using our $\hat{\theta}_{n}$ and $\tilde{\theta}_{n}$ as follows

$$
\begin{aligned}
S_{n}\left(\hat{\theta}_{n}, \tilde{\theta}_{n}\right) & =\sum_{i=1}^{n} \log \frac{f\left(x_{i}, \hat{\theta}_{n}, E^{(i)}\right)}{f\left(x_{i}, \tilde{\theta}_{n}, E^{(i)}\right)} \\
& =\log \frac{\max _{\theta \in \theta} \Pi_{\imath=1}^{n} f\left(x_{i}, \theta, E^{(i)}\right)}{\max _{\varphi \in a\left(\hat{\theta}_{n}\right)} \Pi_{\imath=1}^{n} f\left(x_{i}, \varphi, E^{(i)}\right)} .
\end{aligned}
$$

Definition of mean discrimination. As a measure of discrimination between two probability density functions $f\left(x, \theta, E_{j}\right), f\left(x, \varphi, E_{j}\right)$ we can use by Kullback [4]

$$
I\left(\theta, \varphi, E_{j}\right)=\int_{R}\left[\log \frac{f\left(x, \theta, E_{j}\right)}{f\left(x, \varphi, E_{j}\right)}\right] f\left(x, \theta, E_{j}\right) d x
$$

where $\theta=\left(\theta_{1}, \cdots, \theta_{k}\right), \varphi=\left(\varphi_{1}, \cdots, \varphi_{k}\right)$ and $j=1, \cdots, k .^{1)}$

Existence and uniqueness of $\tilde{\theta}_{n}$. To find $\tilde{\theta}_{n}$, we must minimize $S_{n}\left(\hat{\theta}_{n}, \varphi\right)$ with respect to $\varphi$ in $a\left(\hat{\theta}_{n}\right)$ from the definition of $\tilde{\theta}_{n}$. Since $f\left(x, \theta, E_{j}\right)$ belongs to the exponential family defined in $E_{\jmath}$ we have

so that

$$
\log \frac{\Pi_{\left\{i: E^{(i)}=E j\right]} f\left(x_{i}, \hat{\theta}_{n}, E_{j}\right)}{\Pi_{\left\{i: E^{(i)}=E_{j}\right]} f\left(x_{i}, \varphi, E_{j}\right)}=n_{j} I\left(\hat{\theta}_{n}, \varphi, E_{j}\right)
$$

$$
S_{n}\left(\hat{\theta}_{n}, \varphi\right)=\sum_{j=1}^{k} n_{j} I\left(\hat{\theta}_{n}, \varphi, E_{j}\right) .
$$

Then we must find $\tilde{\theta}_{n}$ on $a\left(\hat{\theta}_{n}\right)$ which minimizes $\sum_{j=1}^{k} n_{j} I\left(\hat{\theta}_{n}, \varphi, E_{j}\right)$ with respect to $\varphi$. First we shall show the fact that $\tilde{\theta}_{n} \in \pi$ for all $n$. But this fact will be given evidently from the property of $\tilde{\theta}_{n}$ minimizing

$$
\sum_{j=1}^{k} n_{j} I\left(\hat{\theta}_{n}, \varphi, E_{j}\right)
$$

on $a\left(\hat{\theta}_{n}\right)$ with respect to $\varphi$, and the convexity of $I\left(\hat{\theta}_{n}, \varphi, E_{j}\right)$ with respect to $\varphi_{\jmath}(j=1, \cdots, k)$ where $\varphi=\left(\varphi_{1}, \cdots, \varphi_{k}\right)$. Therefore we can search $\tilde{\theta}_{n}$ on $\pi$ as to minimizing $S_{n}\left(\hat{\theta}_{n}, \varphi\right)$ with respect to $\varphi$. Put

then

$$
d S_{n}\left(\hat{\theta}_{n}, \varphi\right)=0 \text {, }
$$

$$
\sum_{\jmath=1}^{k} \frac{\partial S_{n}\left(\hat{\theta}_{n}, \varphi\right)}{\partial \varphi_{\jmath}} d \varphi_{\jmath}=\frac{\partial S_{n}\left(\hat{\theta}_{n}, \varphi\right)}{\partial \varphi} \cdot d \varphi=0^{2)}
$$

and we have from $\varphi \in \pi: \mu \cdot \varphi=p$

$$
\mu \cdot d \varphi=0 .{ }^{3)}
$$

1) In this paper we assumed as a restriction of density function of $E_{j}(j=1, \cdots, k)$ that continuity of $d^{2} I\left(\theta, \varphi, E_{j}\right) / d \varphi_{j}^{2}$ with respect to $\varphi_{j}$ for any fixed $\theta_{j}$ in the interval $\Theta_{\jmath}(j=1, \cdots, k)$ respectively.

2) $\partial S_{n}\left(\hat{\theta}_{n}, \varphi\right) / \partial \varphi \cdot d \varphi$ is the innerproduct of two vectors $\partial S_{n}\left({ }_{n}, \varphi\right) / \partial \varphi=\left(\partial S_{n}\left({ }_{n}, \varphi\right) / \partial \varphi_{1}\right.$, $\left.\cdots, \partial S_{n}(\dot{n}, \varphi) / \partial \varphi_{k}\right)$ and $d \varphi=\left(d \varphi_{1}, \cdots, d \varphi_{k}\right)$.

3) $\mu \cdot d \varphi$ is also the innerproduct of the vectors $\mu=\left(\mu_{1}, \cdots, \mu_{k}\right)$ and $d \varphi=\left(d \varphi_{1}, \cdots, d \varphi_{k}\right)$. 
Therefore we get from (2.11), (2.12)

$$
\frac{\partial S_{n}\left(\hat{\theta}_{n}, \varphi\right)}{\partial \varphi}=\text { Constant } \cdot \mu \text {. }
$$

Or equivalently

$$
\frac{n_{1}}{\mu_{1}} \frac{d I\left(\hat{\theta}_{n}, \varphi, E_{1}\right)}{d \varphi_{1}}=\cdots=\frac{n_{k}}{\mu_{k}} \frac{d I\left(\hat{\theta}_{n}, \varphi, E_{k}\right)}{d \varphi_{k}} .
$$

Hence from the relation (2.14) and the convexity of $I\left(\hat{\theta}_{n}, \varphi, E_{j}\right)$ with respect to $\varphi_{\jmath}$, that is, $d I\left(\hat{\theta}_{n}, \varphi, E_{j}\right) / d \varphi_{j}$ is strictly increasing function of $\varphi_{j}(j=1, \cdots, k)$, we can find $\tilde{\theta}_{n}$ on $\pi$ uniquely for any fixed $n$. Therefore if $\hat{\theta}_{n}$ is uniquely given for any fixed $n$ then from (2.14) and $\mu \cdot \varphi=p$ we can find $\tilde{\theta}_{n}$ uniquely on $\pi$ as to be proved.

Lemma 2. Under any sequence $E^{(1)}, \cdots, E^{(n)}$, if $n_{j} \geqq 1(j=1, \cdots, k)$ is satisfied then there exists $\tilde{\theta}_{n}$ uniquely on our hyperplane $\pi$.

Behavior of $\hat{\theta}_{n}$ under any sequence $E^{(1)}, E^{(2)}, \ldots$. Now we shall show the probability equals to zero that $\hat{\theta}_{n}$ does not converge for any sequence $E^{(1)}, E^{(2)}, \cdots$. The event that $\hat{\theta}_{n}$ does not converge is included in the event $\hat{\theta}_{n j}$ does not converge for some $j$. But this event would not occur from the strong law of large numbers. Hence $\hat{\theta}_{n}$ converges with probability 1 for any sequence $E^{(1)}, E^{(2)}, \cdots$.

LEMMA 3. Under any sequence $E^{(1)}, E^{(2)}, \cdots$, our $\hat{\theta}_{n}$ converges with probability 1.

In the following line we put the limit point as $\theta_{0}$ tentatively. Next under any sequence $E^{(1)}, E^{(2)}, \cdots$, we shall prove the probability equals to zero that there exists some integer $N$ such that $\hat{\theta}_{n} \in \pi$ is satisfied for all $n \geqq N$. For any sequence $E^{(1)}, E^{(2)}, \cdots$, there exists some integer $j$ in $1, \cdots, k$ satisfying $n_{j} \rightarrow \infty$ as $n \rightarrow \infty$. And evidently the event that $\hat{\theta}_{n} \in \pi$ for all $n \geqq N$ reduced to the event $\hat{\theta}_{N}=\hat{\theta}_{N+1}=\cdots$, that is, the event that $\hat{\theta}_{N \jmath}=\hat{\theta}_{N+1 \jmath}=\cdots$ for all $j=1, \cdots, k$. But for some $j$ satisfying $n_{j} \rightarrow \infty$ as $n \rightarrow \infty$ the event that the maximum likelihood estimate $\hat{\theta}_{n_{j}}$ gives an identical value for sufficiently large $n$ occurs with probability zero by the zero one law. Hence the event that there exists some integer $N$ such that $\hat{\theta}_{n} \in \pi$ is satisfied for all $n \geqq N$ would not occur at all. So that $\hat{\theta}_{n}$ does not exist on $\pi$ frequentrly $n$ for any sequence $E^{(1)}, E^{(2)}, \cdots$ with probability 1 as to be proved. This property of $\hat{\theta}_{n}$ will play an important part in following section.

\section{$\S 3$. The optimal procedure $\mathscr{L}^{*}$.}

Definition of sequence $\left\{\boldsymbol{\lambda}_{n}\right\}$. In this section, at first we shall define $k$ dimensional ratio vector $\lambda_{n}$ in each step $n$. For fixed $\hat{\theta}_{n}$, we define $\theta_{n}{ }^{*}$ on $\pi$ in subspace of $\Theta$

$$
R_{1} \operatorname{sign} \mu_{1}\left(\hat{\theta}_{n}\right) \otimes \cdots \otimes R_{k} \operatorname{sign} \mu_{k}\left(\hat{\theta}_{n}\right)
$$

where $R_{\jmath}^{-}\left(\hat{\theta}_{n}\right)=\left(-\infty, \hat{\theta}_{n j}\right]$ and $R_{\jmath}^{+}\left(\hat{\theta}_{n}\right)=\left[\hat{\theta}_{n \jmath},+\infty\right)$ such that following equality is satisfied 


$$
\frac{I\left(\hat{\theta}_{n}, \theta_{n}^{*}, E_{1}\right)}{c_{1}}=\cdots=\frac{I\left(\hat{\theta}_{n}, \theta_{n}^{*}, E_{k}\right)}{c_{k}} .
$$

Unique existence of $\theta_{n}{ }^{*}$ for fixed $\hat{\theta}_{n}$ is given by the convexity of $I\left(\hat{\theta}_{n}, \varphi, E_{j}\right)$ with respect to $\varphi_{j}$ for all $j$. If $\hat{\theta}_{n-1} \notin \pi$ then $\hat{\theta}_{n-1} \neq \theta_{n-1} *$. Using this $\hat{\theta}_{n-1}$ and $\theta_{n-1} *$ we shall define $k$ dimensional vector $\lambda_{n}=\left(\lambda_{n 1}, \cdots, \lambda_{n k}\right)$ having $k$ positive components uniquely by the two conditions $\lambda_{n 1}+\cdots+\lambda_{n k}=1$ and

$$
\frac{\lambda_{n 1}}{\mu_{1}}\left[\frac{d I\left(\hat{\theta}_{n-1}, \varphi, E_{1}\right)}{d \varphi_{1}}\right]_{\varphi=\theta_{n-1} *}=\cdots=\frac{\lambda_{n k}}{\mu_{k}}\left[\frac{d I\left(\hat{\theta}_{n-1}, \varphi, E_{k}\right)}{d \varphi_{k}}\right]_{\varphi=\theta_{n-1} *} \cdot{ }^{4)}
$$

And if $\hat{\theta}_{n-1} \in \pi$ then $\hat{\theta}_{n-1}=\theta_{n-1} *$ so we can not find $\lambda_{n}$ uniquely. In this case, we put $\lambda_{n}=\lambda_{n-1}$, moreover we put $\lambda_{1}=(1,0, \cdots, 0), \cdots, \lambda_{k}=(0, \cdots, 0,1)$. In this way we can get $\lambda_{n}$ uniquely for all $n$. Hence $\lambda_{1}, \lambda_{2}, \cdots$ is uniquely defined for any sequence $E^{(1)}, E^{(2)}, \cdots$. In the following line we shall call a vector having all positive components positive vector and denote a positive vector $V$ as $V>0$.

Behavior of $\lambda_{n}$ under any sequence $E^{(1)}, E^{(2)}, \cdots$. Next we shall investigate the behavior of the sequence $\lambda_{n}$. If the limit point $\theta_{0}$ of $\hat{\theta}_{n}$ did not exist on our plane $\pi$ then $\lambda_{n}$ converges to a positive vector $\lambda_{0}=\left(\lambda_{01}, \cdots, \lambda_{0 k}\right)^{5)}$ satisfying $\sum_{\jmath=1}^{k} \lambda_{0 j}=1$ and the next equality analogously as we defined $\lambda_{n}$ uniquely by $\hat{\theta}_{n}$

$$
\frac{\lambda_{01}}{\mu_{1}}\left[\frac{d I\left(\theta_{0}, \varphi, E_{1}\right)}{d \varphi_{1}}\right]_{\varphi=\theta_{0} *}=\cdots=\frac{\lambda_{0 k}}{\mu_{k}}\left[\frac{d I\left(\theta_{0}, \varphi, E_{k}\right)}{d \varphi_{k}}\right]_{\varphi=\theta_{0} *}
$$

where $\theta_{0} *$ is uniquely defined by $\theta_{0}$ on $\pi$ in subspace

$$
R_{1} \operatorname{sign} \mu_{1}\left(\theta_{0}\right) \otimes \cdots \otimes R_{k} \operatorname{sign} \mu_{k}\left(\theta_{0}\right)
$$

satisfying that

$$
\frac{I\left(\theta_{0}, \theta_{0} *, E_{1}\right)}{c_{1}}=\cdots=\frac{I\left(\theta_{0}, \theta_{0} *, E_{k}\right)}{c_{k}} .
$$

So then, under any sequence $E^{(1)}, E^{(2)}, \cdots$, if $\theta_{0}=\lim _{n \rightarrow \infty} \hat{\theta}_{n}$ does not exist on $\pi$, then $\lim _{n \rightarrow \infty} \lambda_{n}=\lambda_{0}>0$ with probability 1 .

Otherwise, if $\theta_{0}=\lim _{n \rightarrow \infty} \hat{\theta}_{n}$ exists on $\pi$, we shall show $\lambda_{n}$ has a positive limit vector with probability 1 , as follows. From the assumption $\lim _{n \rightarrow \infty} \hat{\theta}_{n}=\theta_{0} \in \pi$ we get $\lim _{n \rightarrow \infty} \theta_{n} *=\theta_{0}$ with probability 1 so we have $\lim _{n \rightarrow \infty} \hat{\theta}_{n}=\lim _{n \rightarrow \infty} \theta_{n} *$ and $\lim _{n \rightarrow \infty} \tilde{\theta}_{n}=\theta_{0}$ with probability 1 . By Taylor's expansion

$$
\begin{gathered}
I\left(\hat{\theta}_{n}, \theta_{n}^{*}, E_{j}\right)=\frac{p_{j}\left(\hat{\theta}_{n}-\theta_{n}^{*}\right)^{2}}{2 !}\left[\frac{d^{2} I\left(\hat{\theta}_{n}, \varphi, E_{j}\right)}{d \varphi_{j}{ }^{2}}\right]_{\varphi=\bar{\theta}_{n}}, \\
{\left[\frac{d I\left(\hat{\theta}_{n}, \varphi, E_{j}\right)}{d \varphi_{j}}\right]_{\varphi=\theta_{n}{ }^{*}}=p_{j}\left(\hat{\theta}_{n}-\theta_{n}{ }^{*}\right)\left[\frac{d^{2} I\left(\hat{\theta}_{n}, \varphi, E_{j}\right)}{d \varphi_{j}{ }^{2}}\right]_{\varphi=\overline{\bar{\theta}}_{n}}}
\end{gathered}
$$

4) If $\hat{\theta}_{n-1} \notin \pi$ then we can define $\lambda_{n}$ uniquely by (3.2), where $\theta_{n-1} *$ in (3.2) is defined uniquely by $\hat{\theta}_{n-1}$ from (3.1). Hence we can consider $\lambda_{n}$ as a function of $\hat{e}_{n-1}: \lambda_{n}=\lambda_{n}\left(\hat{\theta}_{n-1}\right)$.

5) Where $\lambda_{0}$ is uniquely given as a function of the limit point $\theta_{0}: \lambda_{0}=\lambda_{0}\left(\theta_{0}\right)$. 
where $p_{j}(\quad)$ is a projection of the vector in $(\quad)$ to $j$-th coordinate, and $\bar{\theta}_{n}, \overline{\bar{\theta}}_{n}$ exists on the intervals

$$
0<p_{j}\left(\hat{\theta}_{n}-\bar{\theta}_{n}\right)<p_{j}\left(\hat{\theta}_{n}-\theta_{n} *\right), \quad 0<p_{j}\left(\hat{\theta}_{n}-\overline{\bar{\theta}}_{n}\right)<p_{j}\left(\hat{\theta}_{n}-\theta_{n} *\right),
$$

respectively, for any fixed $j$. Then $\bar{\theta}_{n}$ and $\overline{\bar{\theta}}_{n}$ converge to $\theta_{0}$ with probability 1 . Therefore

$$
\lim _{n \rightarrow \infty}\left[\frac{d^{2} I\left(\hat{\theta}_{n}, \varphi, E_{j}\right)}{d \varphi_{\jmath}^{2}}\right]_{\varphi=\bar{\theta}_{n}}=\lim _{n \rightarrow \infty}\left[\frac{d^{2} I\left(\hat{\theta}_{n}, \varphi, E_{j}\right)}{d \varphi_{\jmath}^{2}}\right]_{\varphi=\vec{\theta}_{n}}>0 .
$$

So by the definition of $\lambda_{n+1}$ (3.2)

$$
\begin{aligned}
& {\left[\frac{\lambda_{n+12}}{\lambda_{n+1 \jmath}}\right]^{2}=\left[\frac{\mu_{i}}{\mu_{j}}\right]^{2}\left[\frac{d I\left(\hat{\theta}_{n}, \varphi, E_{j}\right)}{d \varphi_{\jmath}}\right]_{\varphi=\theta_{n} *}^{2} /\left[\frac{d I\left(\hat{\theta}_{n}, \varphi, E_{\imath}\right)}{d \varphi_{i}}\right]_{\varphi=\theta_{n}{ }^{*}}^{2}} \\
& =\left[\frac{\mu_{i}}{\mu_{\jmath}}\right]^{2} \frac{p_{j}\left(\hat{\theta}_{n}-\theta_{n}\right)^{2}}{p_{i}\left(\hat{\theta}_{n}-\theta_{n}{ }^{*}\right)^{2}}\left[\frac{d^{2} I\left(\hat{\theta}_{n}, \varphi, E_{j}\right)}{d \varphi_{\jmath}{ }^{2}}\right]_{\varphi=\overline{\bar{\theta}}_{n}}^{2} /\left[\frac{d^{2} I\left(\hat{\theta}_{n}, \varphi, E_{i}\right)}{d \varphi_{i}{ }^{2}}\right]_{\varphi=\bar{\theta}_{n}}^{2} .
\end{aligned}
$$

And by the definition of $\theta_{n}^{*}(3.1)$

$$
\frac{I\left(\hat{\theta}_{n}, \theta_{n}^{*}, E_{\imath}\right)}{c_{\imath}}=\frac{I\left(\hat{\theta}_{n}, \theta_{n}^{*}, E_{j}\right)}{c_{j}} .
$$

Then we have

$$
\frac{p_{j}\left(\hat{\theta}_{n}-\theta_{n}\right)^{2}}{p_{i}\left(\hat{\theta}_{n}-\theta_{n}{ }^{2}\right)^{2}}=\frac{c_{\jmath}}{c_{\imath}}\left[\frac{d^{2} I\left(\hat{\theta}_{n}, \varphi, E_{\imath}\right)}{d \varphi_{i}{ }^{2}}\right]_{\varphi=\bar{\theta}_{n}} /\left[\frac{d^{2} I\left(\hat{\theta}_{n}, \varphi, E_{j}\right)}{d \varphi_{j}{ }^{2}}\right]_{\varphi=\bar{\theta}_{\imath}} .
$$

Therefore from (3.8)

$$
\begin{aligned}
{\left[\frac{\lambda_{n+1 \imath}}{\lambda_{n+1 \jmath}}\right]^{2} } & =\left[\frac{\mu_{i}}{\mu_{\jmath}}\right]^{2} \frac{c_{\jmath}}{c_{\imath}}\left[\frac{d^{2} I\left(\hat{\theta}_{n}, \varphi, E_{i}\right)}{d \varphi_{i}{ }^{2}}\right]_{\varphi=\bar{\theta}_{n}} /\left[\frac{d^{2} I\left(\hat{\theta}_{n}, \varphi, E_{j}\right)}{d \varphi_{\jmath}{ }^{2}}\right]_{\varphi=\bar{\theta}_{n}} \\
& \cdot\left[\frac{d^{2} I\left(\hat{\theta}_{n}, \varphi, E_{j}\right)}{d \varphi_{\jmath}{ }^{2}}\right]_{\varphi=\overline{\bar{\theta}}_{n}}^{2} /\left[\frac{d^{2} I\left(\hat{\theta}_{n}, \varphi, E_{i}\right)}{d \varphi_{i}{ }^{2}}\right]_{\varphi=\overline{\bar{\theta}}_{n}} \cdot
\end{aligned}
$$

So that

$$
\text { (3. 11) } \lim _{n \rightarrow \infty}\left[\frac{\lambda_{n \imath}}{\lambda_{n_{\jmath}}}\right]^{2}=\left[\frac{\mu_{i}}{\mu_{\jmath}}\right]^{2} \frac{c_{\jmath}}{c_{\imath}}\left[\frac{d^{2} I\left(\theta_{0}, \varphi, E_{j}\right)}{d \varphi_{\jmath}{ }^{2}}\right]_{\varphi=\theta_{0}} /\left[\frac{d^{2} I\left(\theta_{0}, \varphi, E_{\imath}\right)}{d \varphi_{i}{ }^{2}}\right]_{\varphi=\theta_{0}} \text {. }
$$

Since the right hand limit value is positive, our sequence $\lambda_{n}$ has a positive limit vector with probability 1 , we put the vector as $\boldsymbol{\lambda}_{0}$ tentatively. As a conclusion we get in any sequence $E^{(1)}, E^{(2)}, \cdots$ there exists a positive vector $\lambda_{0}$ such that $\lim _{n \rightarrow \infty} \boldsymbol{\lambda}_{n}=\boldsymbol{\lambda}_{0}>0$ is satisfied with probability 1 .

LEMMA 4. Under any sequence $E^{(1)}, E^{(2)}, \cdots, \lambda_{n}$ converges to a positive vector as $n \rightarrow \infty$ with probability 1 .

The optimal procedure $\mathscr{L}^{*}$. Using the sequence $\lambda_{n}(n \geqq 1)$ defined above, we 
consider $E^{(n)}(n \geqq 1)$ as a sequence of random variables which take values $E_{1}, \cdots, E_{k}$ in each step $n$ and have probabilities as follows, for each $n \geqq 1$

$$
\text { Prob }\left\{E^{(n)}=E_{\jmath}\right\}=\lambda_{n \jmath} \text {. }
$$

In the following line we call this randomized policy procedure $\mathscr{L}^{*}$.

Proprety of our procedure $\mathscr{L}^{*}$. By Lemma 4 we observed the fact that in any sequence $E^{(1)}, E^{(2)}, \cdots$ there exists a positive vector $\lambda_{0}$ such that $\lambda_{n}$ converges to $\lambda_{0}$. Using this fact we get under our procedure $\mathscr{L}^{*} n_{j} / n$ converges to $j$-th component $\lambda_{0 j}(>0)$ of the limit vector $\lambda_{0}$ by the strong law of large numbers. Hence under our procedure $\mathscr{L}^{*} n_{j} \rightarrow \infty$ as $n \rightarrow \infty$ for any $j=1, \cdots, k$ is satisfied so that $\hat{\theta}_{n}$ converges to the unknown true parameter $\theta$ as to be proved.

THEOREM 1. Under our procedure $\mathscr{L}^{*}, \hat{\theta}_{n}$ converges to the unknown true parameter $\theta$ as $n \rightarrow \infty$ with probability 1.

Next we suppose the unknown true parameter $\theta$ is not an element of $\pi$. Then there exists a positive vector $\lambda$ uniquely given by $\theta$, as we defined $\lambda_{n}$ uniquely by $\hat{\theta}_{n-1}$ and $\lambda_{0}$ uniquely by $\theta_{0}$, such that $\lim _{n \rightarrow \infty} \lambda_{n}=\lambda$ is satisfied with probability 1 . This fact is shown as discussion of Lemma 4 . Where the vector $\lambda=\left(\lambda_{1}, \cdots, \lambda_{k}\right)$ is defined satisfying $\sum_{\jmath=1}^{k} \lambda_{\jmath}=1$ and analogousely as (3.3)

$$
\frac{\lambda_{1}}{\mu_{1}}\left[\frac{d I\left(\theta, \varphi, E_{1}\right)}{d \varphi_{1}}\right]_{\varphi=\theta^{*}}=\cdots=\frac{\lambda_{k}}{\mu_{k}}\left[\frac{d I\left(\theta, \varphi, E_{k}\right)}{d \varphi_{k}}\right]_{\varphi=\theta^{*}}
$$

where $\theta^{*}$ is uniquely defined by $\theta$ on $\pi$ in subspace

$$
R_{1} \operatorname{sign} \mu_{1}(\theta) \otimes \cdots \otimes R_{k} \operatorname{sign} \mu_{k}(\theta)
$$

satisfying that analogously as (3.4)

$$
\frac{I\left(\theta, \theta^{*}, E_{1}\right)}{c_{1}}=\cdots=\frac{I\left(\theta, \theta^{*}, E_{k}\right)}{c_{k}} .
$$

Otherwise if the unknown true parameter $\theta$ is an element of $\pi$, then under our procedure $\mathcal{L}^{*}$, from the result of Lemma $4, \lambda_{n}$ converges to a positive vector with probability 1 . Hence under our procedure $\mathscr{L}^{*}$ if $\theta \notin \pi$ then $n_{j} / n$ converges to $\lambda_{j}(j=1, \cdots, k)$ with probability 1 , and if $\theta \in \pi$ then $n_{j} / n$ converges to a positive value with probability 1 as to be proved.

COROLlary 1. Under our procedure $\mathscr{L}^{*}$, if the true unknown parameter $\theta$ is not an element of $\pi$ then $\left(n_{1} / n, \cdots, n_{k} / n\right)$ converges to our vector $\lambda(>0)$, defined in (3.13), is satisfied with probability 1 , and otherwise if the true unknown parameter $\theta$ is an element of $\pi$ then $\left(n_{1} / n, \cdots, n_{k} / n\right)$ converges to a positive vector is satisfied with probability 1.

Optimal condition and optimal ratio vector. As a conclusion of Corollary 1 , we have shown under our procedure $\mathscr{L}^{*}$ that if $\theta \notin \pi$, then $n_{j} / n \rightarrow \lambda_{j}$ with probability 1 , 
and otherwise if $\theta \in \pi$, then $n_{j} / n$ converges to a positive value $(j=1, \cdots, k)$ with probability 1 . In the following line we call this proposition as optimal condition and the vector $\lambda$ defined in $(3.13)$ as optimal ratio vector. The meaning of optimality will be given in following main theorems of next section.

\section{$\S 4$. Main theorems and the proofs.}

Under the optimal condition given in the preceding section, if the true unknown parameter $\theta$ is not an element of $\pi$, then we get $\lim _{n \rightarrow \infty} \tilde{\theta}_{n}=\theta^{*}$ with probability 1 , from the two equalities (2.14), (3.13)

$$
\begin{aligned}
& \frac{n_{1}}{\mu_{1}}\left[\frac{d I\left(\hat{\theta}_{n}, \varphi, E_{1}\right)}{d \varphi_{1}}\right]_{\varphi=\tilde{\theta}_{n}}=\cdots=\frac{n_{k}}{\mu_{k}}\left[\frac{d I\left(\hat{\theta}_{n}, \varphi, E_{k}\right)}{d \varphi_{k}}\right]_{\varphi=\tilde{\theta}_{n}}, \\
& \frac{\lambda_{1}}{\mu_{1}}\left[\frac{d I\left(\theta, \varphi, E_{1}\right)}{d \varphi_{1}}\right]_{\varphi=\theta^{*}}=\cdots=\frac{\lambda_{k}}{\mu_{k}}\left[\frac{d I\left(\theta, \varphi, E_{k}\right)}{d \varphi_{k}}\right]_{\varphi=\theta^{*}} .
\end{aligned}
$$

So we get

$$
\lim _{n \rightarrow \infty} \frac{I\left(\hat{\theta}_{n}, \tilde{\theta}_{n}, E_{j}\right)}{c_{\jmath}}=I^{*}(\theta)
$$

with probability 1 , where $I^{*}(\theta)$ is the value of (3.14)

$$
I^{*}(\theta)=\frac{I\left(\theta, \theta^{*}, E_{1}\right)}{c_{1}}=\cdots=\frac{I\left(\theta, \theta^{*}, E_{k}\right)}{c_{k}} .
$$

And from the definitions of $S_{n}\left(\hat{\theta}_{n}, \tilde{\theta}_{n}\right)$ and $\sum_{\imath=1}^{n} C^{(i)}$

$$
\frac{S_{n}\left(\hat{\theta}_{n}, \tilde{\theta}_{n}\right)}{\sum_{\imath=1}^{n} C^{(i)}}=\frac{n_{1} I\left(\hat{\theta}_{n}, \tilde{\theta}_{n}, E_{1}\right)+\cdots+n_{k} I\left(\hat{\theta}_{n}, \tilde{\theta}_{n}, E_{k}\right)}{n_{1} c_{1}+\cdots+n_{k} c_{k}} .
$$

Hence the sum of information relative to the sum of costs for the first $n$ trials to discriminate $\mu \cdot \theta>p$ or not

$$
\frac{S_{n}\left(\hat{\theta}_{n}, \tilde{\theta}_{n}\right)}{\sum_{\imath=1}^{n} C^{(i)}}
$$

converges to the value $I^{*}(\theta)$ with probability 1 as to be proved. And otherwise if the true unknown parameter $\theta$ is an element of $\pi$, then we get $\lim _{n \rightarrow \infty} \tilde{\theta}_{n}=\theta$ with probability 1 . So we get

$$
\lim _{n \rightarrow \infty} \frac{I\left(\hat{\theta}_{n}, \tilde{\theta}_{n}, E_{j}\right)}{c_{\jmath}}=0
$$

with probability 1 . Hence our

$$
\frac{S_{n}\left(\hat{\theta}_{n}, \tilde{\theta}_{n}\right)}{\sum_{\imath=1}^{n} C^{(i)}}
$$


converges to zero with probability 1 as to be proved.

THEOREM 2. Under our optimal condition if the true unknown parameter $\theta$ is not an element of $\pi$ then we get

$$
\lim _{n \rightarrow \infty} \frac{S_{n}\left(\hat{\theta}_{n}, \tilde{\theta}_{n}\right)}{\sum_{\imath=1}^{n} C^{(i)}}=I^{*}(\theta)
$$

with probability 1, and otherwise if the true unknown parameter $\theta$ is an element of $\pi$ then we get

$$
\frac{S_{n}\left(\hat{\theta}_{n}, \tilde{\theta}_{n}\right)}{\sum_{\imath=1}^{n} C^{(i)}}
$$

converges to zero with probability 1.

By Corollary 1 we have shown that our procedure $\mathscr{L}^{*}$ has a property of optimal condition. Therefore we have next corollary.

COROLlaRY 2. Under our procedure $\mathscr{L}^{*}$ we get the same result as given in Theorem 2.

Meaning of optimality. In the following line we consider a class of procedure satisfying $\min _{j}\left(n_{j}\right) \rightarrow \infty$ as $n \rightarrow \infty$, and $\left(n_{1} / n, \cdots, n_{k} / n\right)$ converges to vector $\lambda^{\prime}=\left(\lambda_{1}{ }^{\prime}, \cdots, \lambda_{k}{ }^{\prime}\right)$ where $\sum_{j=1}^{k} \lambda_{j}{ }^{\prime}=1$. In this class of procedure we shall show that our procedure $\mathscr{L}^{*}$ is asymptotically most informative one relative to the sum of costs to discriminate $\mu \cdot \theta<p$ or not. That is, under another procedure $\mathscr{L}^{* *}$ having the limit ratio $\lambda^{\prime}$ different from our ratio $\lambda$ we can get asymptotically less information relative to the sum of costs to discriminate $\mu \cdot \theta>p$ or not than we get using our procedure $\mathscr{L}^{*}$. Under the procedure $\mathscr{L}^{* *}$, fixed by the limit ratio $\lambda^{\prime}$ different from our ratio $\lambda$, how much information to discriminate $\mu \cdot \theta>p$ or not we can get asymptotically relative to the sum of costs? From the first condition that $\min _{j}\left(n_{j}\right) \rightarrow \infty$ as $n \rightarrow \infty$ we get $\lim _{n \rightarrow \infty} \hat{\theta}_{n}=\theta$ with probability 1 . If we assume the true parameter $\theta$ does not exist on $\pi$ then for a given ratio in the second condition: $\left(n_{1} / n, \cdots, n_{k} / n\right)$ converges to ratio $\lambda^{\prime}=\left(\lambda_{1}{ }^{\prime}, \cdots, \lambda_{k}{ }^{\prime}\right)$ as $n \rightarrow \infty\left(\lambda^{\prime} \neq \lambda\right)$, we can define $\theta^{* *}$ uniquely as an element of $\pi$ and satisfying

$$
\frac{\lambda_{1}^{\prime}}{\mu_{1}}\left[\frac{d I\left(\theta, \varphi, E_{1}\right)}{d \varphi_{1}}\right]_{\varphi=0^{* *}}=\cdots=\frac{\lambda_{k}^{\prime}}{\mu_{k}}\left[\frac{d I\left(\theta, \varphi, E_{k}\right)}{d \varphi_{k}}\right]_{\varphi=\theta^{* *}} .
$$

From the equality (2.14) defining $\tilde{\theta}_{n}$ uniquely on our hyperplane $\pi$ we can verify $\lim _{n \rightarrow \infty} \tilde{\theta}_{n}=\theta^{* *}$ with probability 1 . Therefore

$$
\lim _{n \rightarrow \infty} \frac{S_{n}\left(\hat{\theta}_{n}, \tilde{\theta}_{n}\right)}{\sum_{r=1}^{n} C^{(i)}}=\frac{\lambda_{1}^{\prime} I\left(\theta, \theta^{* *}, E_{1}\right)+\cdots+\lambda_{k}^{\prime} I\left(\theta, \theta^{* *}, E_{k}\right)}{\lambda_{1}^{\prime} C_{1}+\cdots+\lambda_{k}^{\prime} c_{k}}
$$

is satisfied with probability 1 . So we denote the limit value under the procedure 
$\mathscr{L}^{* *}$ as $I^{* *}(\theta)$ in the following line. From the inequality $\lambda^{\prime} \neq \lambda$ in the second condition we have $\theta^{* *} \neq \theta^{*}$ so we get the next inequality

$$
\lambda_{1}{ }^{\prime}\left(\theta, \theta^{* *}, E_{1}\right)+\cdots+\lambda_{k}{ }^{\prime} I\left(\theta, \theta^{* *}, E_{k}\right)<\lambda_{1}{ }^{\prime} I\left(\theta, \theta^{*}, E_{1}\right)+\cdots+\lambda_{k}{ }^{\prime} I\left(\theta, \theta^{*}, E_{k}\right) .
$$

Therefore we have

$$
I^{* *}(\theta)<I^{*}(\theta)
$$

Otherwise if the true parameter $\theta$ is an element of $\pi$ then we have $\lim _{n \rightarrow \infty} \tilde{\theta}_{n}=\theta$ with probability 1 . So that

$$
\frac{S_{n}\left(\hat{\theta}_{n}, \tilde{\theta}_{n}\right)}{\sum_{\imath=1}^{n} C^{(i)}}
$$

converges to zero with probability 1 as to be proved.

THEOREM 3. Under any procedure $\mathscr{L}^{* *}$, satisfying $\min _{j}\left(n_{j}\right) \rightarrow \infty$ as $n \rightarrow \infty$ and $\left(n_{1} / n, \cdots, n_{k} / n\right)$ converges to $\lambda^{\prime}=\left(\lambda_{1}{ }^{\prime}, \cdots, \lambda_{k}{ }^{\prime}\right)$ as $n \rightarrow \infty\left(\lambda^{\prime} \neq \lambda\right)$, if the true unknown parameter $\theta$ is not an element of $\pi$ then we get

$$
\lim _{n \rightarrow \infty} \frac{S_{n}\left(\hat{\theta}_{n}, \tilde{\theta}_{n}\right)}{\sum_{\imath=1}^{n} C^{(i)}}=I^{* *}(\theta)<I^{*}(\theta)
$$

is satisfied with probability 1. And otherwise if the true unknown parameter $\theta$ is an element of $\pi$ then

$$
\frac{S_{n}\left(\hat{\theta}_{n}, \tilde{\theta}_{n}\right)}{\sum_{\imath=1}^{n} C^{(i)}}
$$

converges to zero with probability 1.

Having expected the meaning of Theorem 3 we have called the property of our procedure $\mathscr{L}^{*}$ as optimal condition or having optimal ratio vector.

\section{$\S 5$. Original procedure $\mathscr{L}$ in the case $k=2$.}

Original procedure $\mathscr{L}$. We consider two exponential trials $E_{1}, E_{2}$ and use the procedure $\mathscr{L}$ given in [2], [3] that is $E^{(1)}=E_{1}, E^{(2)}=E_{2}$ and for $n \geqq 2$ we define successively

$$
E^{(n+1)} \in\left\{E: \text { which maximizes } \frac{I\left(\hat{\theta}_{n}, \tilde{\theta}_{n}, E_{i}\right)}{c_{\imath}} \quad(i=1,2)\right\}
$$

Under this procedure $\mathscr{L}$ we shall show the optimal condition. Following to Lemma 3 , under any sequence we have $\hat{\theta}_{n}$ converges with probability 1 . By Lemma $4 \lambda_{n}$ converges to a positive ratio vector with probability 1 . And also the procedure $\mathscr{L}$ has a property $n_{j} / n-\lambda_{n}$ converges to zero as $n \rightarrow \infty$ with probability 1 as the proof 
given in our paper [2]. Therefore $n_{j} / n$ converges to a positive value with probability 1 . So that under the procedure $\mathscr{L}$ we have $\min \left(n_{1}, n_{2}\right) \rightarrow \infty$ as $n \rightarrow \infty$ with probability 1 . Hence under the procedure $\mathscr{L}$ we have $\hat{\theta}_{n} \rightarrow \theta$ as $n \rightarrow \infty$ with probability 1 . If the true parameter $\theta$ is not an element of $\pi$ then $\lambda_{n}$ converges to $\lambda$ with probability 1 . Then $n_{j} / n$ converges to $\lambda_{j}$ with probability 1 . And otherwise $n_{j} / n$ converges to a positive value with probability $1(j=1,2)$ respectively. Therefore we can verify the procedure $\mathscr{P}$ also have the optimal condition as to be proved. Then by Theorem 2 we get the limit equality (4.2) with probability 1 . Hence this procedure $\mathscr{L}$ also has the optimal property, that is, most informative relative to the sum of costs than any other procedure having the two conditions $n_{j} \rightarrow \infty$ as $n \rightarrow \infty,\left(n_{1} / n, n_{2} / n\right)$ converges to $\lambda^{\prime}=\left(\lambda_{1}{ }^{\prime}, \lambda_{2}{ }^{\prime}\right)$ as $n \rightarrow \infty\left(\lambda^{\prime} \neq \lambda\right)$.

Example of the case $k=2$. We consider two trials $E_{1}, E_{2}$ depending normal distributions with unknown means and known variances respectively. Then the density functions of $E_{1}, E_{2}$ are expressed as follows

$$
f\left(x, \theta, E_{j}\right)=\frac{1}{\sqrt{2 \pi} \sigma_{\jmath}} \exp \left\{-\frac{\left(x-m_{j}\right)^{2}}{2 \sigma_{\jmath}^{2}}\right\} \quad(j=1,2)
$$

where $\theta=\left(m_{1}, m_{2}\right)$ is the pair of unknown means, then we get

$$
I\left(\theta, \varphi, E_{j}\right)=\frac{\left(m_{j}-m_{j}^{*}\right)^{2}}{2 \sigma_{j}^{2}} \quad(j=1,2)
$$

where $\theta=\left(m_{1}, m_{2}\right), \varphi=\left(m_{1}{ }^{*}, m_{2}{ }^{*}\right)$. And the subspaces $H_{1}, H_{2}$ and $\pi$ are given by $\mu=\left(\mu_{1}, \mu_{2}\right)$ and $p$, that is

$$
H_{1}=\left\{\theta: \mu_{1} m_{1}+\mu_{2} m_{2}>p\right\}, \quad H_{2}=\left\{\theta: \mu_{1} m_{1}+\mu_{2} m_{2}<p\right\},
$$

$$
\pi=\left\{\theta: \mu_{1} m_{1}+\mu_{2} m_{2}=p\right\} .
$$

And $\lim _{n \rightarrow \infty} \tilde{\theta}_{n}=\theta^{*}$ is given uniquely by the equality (3.14) and $\theta^{*} \in \pi$.

$$
\frac{\left(m_{1}-m_{1}^{*}\right)^{2}}{2 c_{1} \sigma_{1}^{2}}=\frac{\left(m_{2}-m_{2}^{*}\right)^{2}}{2 c_{2} \sigma_{2}^{2}}
$$

where $\theta=\left(m_{1}, m_{2}\right)$ is the true parameter and $\theta^{*}=\left(m_{1}^{*}, m_{2}^{*}\right)$ is the limit point of $\tilde{\theta}_{n}$. And the limit value of (4.2) $I^{*}(\theta)$ is given as the value of (5.5). Moreover we put $\mu_{1}=1 / \sqrt{2}, \mu_{2}=-1 / \sqrt{2}$ and $p=0$ then our subspaces become as following

$$
\begin{gathered}
H_{1}=\left\{\left(m_{1}, m_{2}\right): m_{1}>m_{2}\right\}, \quad H_{2}=\left\{\left(m_{1}, m_{2}\right): m_{1}<m_{2}\right\}, \\
\pi=\left\{\left(m_{1}, m_{2}\right): m_{1}=m_{2}\right\} .
\end{gathered}
$$

In this case we can get the optimal ratio $\lambda$ as following by (3.13)

$$
\frac{\lambda_{1}\left(m_{1}-m_{1}^{*}\right)}{\sigma_{1}^{2}}=-\frac{\lambda_{2}\left(m_{2}-m_{2}^{*}\right)}{\sigma_{2}^{2}}
$$

Therefore from the two relations (5.5), (5.7) we get the optimal ratio $\lambda$ as next 
value uniformly on $\Theta .^{6)}$

$$
\lambda=\left(\frac{\sqrt{c_{2}} \sigma_{1}}{\sqrt{c_{1}} \sigma_{2}+\sqrt{c_{2}} \sigma_{1}}, \quad \frac{\sqrt{c_{1}} \sigma_{2}}{\sqrt{c_{1}} \sigma_{2}+\sqrt{c_{2}} \sigma_{1}}\right)
$$

Here we additionally suppose ${\sigma_{1}}^{2}={\sigma_{2}}^{2}$, then the optimal ratio $\lambda$ becomes

$$
\lambda=\left(\frac{\sqrt{c_{2}}}{\sqrt{c_{1}}+\sqrt{c_{2}}}, \frac{\sqrt{c_{1}}}{\sqrt{c_{1}}+\sqrt{c_{2}}}\right)
$$

We can see this result in our paper [3], that is, this result of Section 5 is a generalization of papers [2], [3] as to be proved.

Acknowledgement. The auther expresses hearty thanks to Professor $\mathrm{K}$. Kunisawa for many useful suggestions.

\section{REFERENCES}

[1] Chernoff, H., Sequential design of experiments. Ann. Math. Stat. 30 (1959), 755-770.

[2] Kawamura, K., Asymptotic behavior of sequential design with costs of experiments. Kōdai Math. Sem. Rep. 16 (1964), 169-182.

[3] Kawamura, K., Asymptotic behavior of sequential design with costs of experiments (The case of normal distribution). Kōdai Math. Sem. Rep. 17 (1965), 48-52.

[4] Kullback, S., Information theory and statistics. (1959), Wiley.

[5] Kunisawa, K., Modern probability theory. 12th Ed. (1963), Iwanami Co. (In Japanese)

[6] KunisawA, K., Introduction to information theory for operations research. 4th Ed. (1963), J. U. S. E. (In Japanese)

6) Generally $\lambda$ is a function of $\theta$ on $\Theta(\theta \notin \pi)$ but, in this special example of trials $E_{1}, E_{2}$ with normal distributions of the case $k=2, \lambda$ does not depending on $\theta$, that is, $\lambda$ equals to a constant value uniformly on $\Theta(\theta \notin \pi)$.

This is the reason why, in this case of special example, the optimal procedure $\mathscr{P}$ does not depending on previous observations till now but only on sample sizes till now as we showed in the note in [3]. 